\title{
A Avaliação Crítica da Literatura Médica como Instrumento de Complementação Educacional no Internato de Medicina
}

\section{The Critical Evaluation of Medical Literature as an Educational Supplementary Tool in Medical Internship}

Francisco Eduardo Silva ${ }^{I}$

\section{PALAVRAS-CHAVE}

- Estudantes de Medicina.

- Medicina Baseada em Evidência.

- Metodologia.

- Artigo de Revista.

\section{RESUMO}

Este artigo aborda a literatura científica como um instrumento de auxílio na busca do conhecimento pelo interno de Medicina, associada a uma avaliação crítica de publicações de artigos médicos. A produção científica no mundo tem se tornado um grande aliado do ensino médico e pode ser intensificada no internato de Medicina. A saúde baseada em evidência, que engloba a melhor literatura científica, associada à experiência clínica e à vontade do paciente, tem despertado a necessidade do profissional de saúde de se atualizar por meio da literatura médica. Entretanto, muitos artigos científicos estão sendo produzidos e publicados sem terem sua qualidade avaliada. Saber escolher uma literatura por meio do conhecimento de sua crítica auxilia o profissional de saúde a otimizar seu tempo. Na graduação, o estudante tem espaço para aprender a identificar fatores relevantes para definir qual o melhor artigo e, juntamente com o aprendizado clínico, entender e discutir a melhor conduta para o paciente. A pergunta é o ponto de partida do estudante de graduação, seguida pela busca da melhor terapia, dos melhores exames diagnósticos, qualidade de vida do paciente e prognóstico de alguma doença. Esse universo impulsiona o aluno de graduação a se interessar pela leitura de um artigo relacionado a sua dúvida. A facilidade de realizar buscas nos bancos de dados eletrônicos auxilia na escolha da melhor literatura e traz ao leitor o que está sendo produzido no mundo científico. O aprendizado de como avaliar criticamente a literatura científica tem se tornado um grande parceiro do aluno da graduação de Medicina na busca de novos conhecimentos com evidência científica de alta qualidade. A importância da avaliação crítica da literatura reside em despertar nos estudantes o interesse pela pesquisa clínica associada ao conhecimento de novas tecnologias produzidas mundialmente. Sua aplicação consciente possibilita melhor qualidade na assistência clínica. 


\section{KEY-WORDS}

- Students, Medical.

- Evidence-Based Medicine.

- Methodology.

- Journal Article.

Recebido em 7/12/2016

Aceito em 7/11/2017

\section{ABSTRACT}

This article discusses the scientific literature as an instrument that helps the search of new knowledge for Internship combined with an assessment of critical medical articles publications. The scientific production around the world has become a great ally in medical education can be intensified in the medical internship. Evidence-based health includes the best scientific literature associated with clinical expertise and the patient's will. Evidence-based health it has aroused the need for the health care professional to upgrade through the medical literature, but many scientific literatures are being produced and published without quality evaluated. Knowing how to choose a literature through knowledge of his criticism helps the health care professional to optimize your time. It is in medical schools where the student has the place to learn to identify relevant factors to determine the best product and together with clinical learning to understand and discuss the best management of the patient. The question is the basis of the undergraduate student, followed by the search for better therapy, diagnostic exams, patient's quality of life and the prognosis of diseases. This universe is the thrust of undergraduate students interested in reading an article related to your question. The ease of searches in electronic databases helps in the best literature choice and brings the reader what is being produced by the scientific world. Learning how to critically evaluate scientific literature has become a major partner of undergraduate students in the search for new knowledge with scientific evidence of high quality. The importance in internship teaching of the critical evaluation of literature is to awake on us the interest in clinical research associated with knowledge of new technologies produced around the world, its application consciously to get a better quality of clinical care.

Aceito em $7 / 11 / 2017$

\section{INTRODUÇÃO}

Na era da saúde baseada em evidência, uma das habilidades importantes que um médico necessita adquirir é a capacidade de analisar criticamente uma literatura científica. Inicialmente, a busca nos bancos de dados eletrônicos disponíveis na internet é uma forma prática e barata de adquirir ensaios clínicos e publicações em periódicos de qualidade, assim como suas publicações. A prática da Medicina Baseada em Evidência abrange um contexto em que a experiência clínica é integrada com a capacidade de analisar criticamente a literatura científica sobre determinado assunto e ajustar o interesse do paciente em tratamento para aplicá-la em prol da melhoria da qualidade da assistência médica ${ }^{1}$.

A evidência científica hoje é um dos fundamentos mais significativos na prática médica, e a Medicina Baseada em Evidência ganhou espaço importante na graduação médica. A leitura crítica de um artigo científico ganha cada vez mais espaço no ensino médico, assim como em outras áreas acadêmicas. A medicina, nos dias atuais, tem sido alvo de grande e rápida renovação tecnológica, transformando o médico em um profissional que precisa estar em constante atualização. Essa transformação em grande escala está sendo sentida nas escolas médicas, dentro das salas de aula e na prática diária do estudante. Nesse cenário, as publicações médicas de estudos científicos em periódicos, sejam artigos de revisão, ensaios clínicos ou pesquisas clínicas, têm auxiliado estudantes no decorrer da graduação médica² . O artigo publicado em um periódico mostra o que está acontecendo no mundo científico, fazendo com que seu leitor identifique trabalhos que podem auxiliá-lo em sua prática diária ou ajudá-lo a esclarecer dúvidas sobre alguma patologia ou caso clínico. Assim, a leitura crítica da literatura médica é um instrumento de auxílio importante para o estudante de graduação.

Na atualidade, os cursos de Medicina, por meio de seu conteúdo programático, visam desenvolver no estudante seu potencial intelectual, sua capacidade de análise e julgamento, assim como uma avaliação crítica comum à habilidade de resolver problemas de forma inquiridora. No cenário atual da formação médica, as informações que chegam a cada dia sobre novas técnicas de tratamento e diagnóstico e novos medicamentos, sob a forma de artigos científicos, além de auxiliarem nessa formação, têm trazido questionamentos sobre o que pode ou não ser feito no campo da prática médica e naquilo que é lido nas publicações recentes nos bancos de dados de pesquisa clínica. 
O interno de Medicina, por estar mais próximo do campo prático devido às suas atividades curriculares estabelecidas pelas escolas médicas, tem no canal teórico um local onde questionamentos podem ser respondidos com o aprendizado de como avaliar criticamente a literatura médica, a fim de escolher a melhor evidência científica publicada ${ }^{2,3}$.

\section{A CRÍTICA DA LITERATURA CIENTÍFICA COMO FERRAMENTA USADA NO ÂMBITO DA GRADUAÇÃO MÉDICA}

Atualmente, muitos periódicos científicos na área biomédica são publicados, porém nem todas as informações contidas são confiáveis. Existem cerca de 2 milhões de artigos científicos publicados na área de saúde, mas apenas $5 \%$ podem ser aproveitados ou são reproduzíveis. A leitura crítica da literatura científica está se tornando cada vez mais importante devido à abundância de artigos publicados em revistas especializadas. A partir do século XX, as informações se expandiram, e o acesso a elas foi facilitado principalmente com o advento da internet e dos bancos de dados eletrônicos, com rapidez e baixo custo. Existe hoje uma grande necessidade de uma leitura crítica da literatura médica pelo estudante de graduação em Medicina, principalmente no internato, que é um período de prática médica com características especiais, no qual a assistência, muitas vezes, pode estar associada a dúvidas sobre condutas diante de um caso clínico específico.

O conhecimento da epidemiologia clínica e da metodologia contida na literatura científica é necessário para o julgamento crítico dos artigos publicados e para sua aplicação na prática médica diária. $\mathrm{O}$ raciocínio científico do estudante de Medicina, a capacidade de pesquisar novas fontes de informações, avaliar a qualidade dessas informações e a força das evidências, favorecendo ou negando o valor de determinada conduta, fazem com que ele chegue a conclusões mais corretas.

A aplicação das conclusões dessa avaliação com a intenção de melhorar os cuidados prestados aos pacientes é o objetivo final do ensino da avaliação crítica da literatura médica. O internato médico é um espaço para se desenvolver a prática médica com uma tutoria presente, que possa ajudar nas atividades práticas e discutir qualquer aprofundamento teórico, como no caso de artigos científicos que vão complementar o raciocínio clínico do estudante frente a um problema ${ }^{4}$.

A leitura regular de revistas especializadas é uma forma eficaz de se manter atualizado. Antes de iniciar a leitura de um artigo científico, o interno já deve ter uma pergunta clara e objetiva com a intenção de obter informações precisas sobre determinado assunto. Na graduação médica, o leitor identifica o problema - se é uma intervenção ou um teste de acurácia - e busca soluções ou orientações na literatura médica, mas, para isso, são necessárias ferramentas que o auxiliem a entender como agir. Contudo, tendo em vista que o internato é o ciclo final do curso de graduação em Medicina, livre de disciplinas acadêmicas e durante o qual o estudante deve receber treinamento intensivo prático e contínuo, sob supervisão, muitas vezes o aprendizado sobre avaliação da literatura científica fica prejudicado.

A avaliação crítica dos estudos, entretanto, não depende somente da habilidade do profissional em interpretar seus resultados ou entender sua metodologia, mas também da maneira como os pesquisadores e responsáveis por sua divulgação descrevem as informações. O relato incompleto ou inadequado de informações sobre o planejamento e a condução dos estudos prejudica a identificação de possíveis erros metodológicos, dificultando o uso de suas conclusões pelos interessados, uma vez que estes não conseguem avaliar criticamente sua aplicabilidade clínica.

Para avaliar a qualidade das informações publicadas, é importante conhecer a metodologia de pesquisa, e é nesse aspecto que entra o docente, incentivando o interno a pesquisar nos bancos de dados. É fundamental que o professor auxilie o aluno a adquirir conhecimento de bioestatística para que este possa entender o objetivo do estudo, identificar a metodologia empregada e avaliar os resultados obtidos a fim de que possa aplicá-los ou não no campo prático.

A formulação da pergunta é o primeiro passo para o interno de Medicina iniciar sua pesquisa no banco de dados eletrônicos. Muitas vezes, a busca vai depender de filtros importantes que o aluno deve aprender durante seu treinamento com o preceptor ou docente. O problema que necessita de uma resposta depende do conhecimento do aluno sobre identificar a qualidade das evidências dos estudos epidemiológicos adquiridos nos programas teóricos nas salas de aula durante a graduação médica ou no período do internato.

O importante para o interno na avaliação crítica da literatura médica após sua busca e identificação de um estudo ou de estudos para pesquisa é se há questões científicas interessantes e que, possivelmente, possam responder à sua pergunta. $\mathrm{O}$ treinamento do aluno em avaliar um estudo deve conter uma identificação metodológica satisfatória. As ferramentas usadas pelos internos na avaliação crítica da literatura podem variar de acordo com o estudo da amostra usada pelo pesquisador, se o artigo escolhido é inovador ou se existe validade interna e validade externa. Na leitura de um artigo científico, o aluno deverá ser orientado a avaliar inicialmente o título do estudo e identificar se há coerência com a conclusão ou se responde à pergunta inicial. $\mathrm{O}$ risco de vieses e a identificação 
das variáveis independentes usadas pelo autor são de grande importância na identificação de um bom estudo.

A prática de introduzir a avaliação crítica da literatura no internato de Medicina visa trazer para o aluno não só o interesse pela pesquisa e o que está acontecendo no mundo científico, como também usar as informações obtidas na sua prática clínica ${ }^{5}$. Ao avaliar criticamente um artigo científico, o aluno não precisa aceitar a conclusão do autor de maneira direta, podendo questionar e elaborar suas próprias conclusões ${ }^{6}$.

Uma questão importante quando se avalia criticamente um artigo científico publicado é o fator de impacto do periódico e dos autores. As revistas científicas apresentam exigências para a publicação que, muitas vezes, podem ser identificadas pelos leitores. Procurar o fator de impacto do periódico onde o artigo foi publicado pode ser uma forma importante para a escolha do artigo, assim como identificar outras publicações do autor ou autores. Os bancos de dados eletrônicos, como PubMed, Embase, Lilacs e Cochrane Library, podem ser usados como referência dos autores, assim como a avaliação de outros trabalhos e artigos publicados.

O tipo de pergunta a ser respondida no questionamento do leitor que busca a literatura científica deve ser adaptado ao tipo de problema. O direcionamento da pesquisa nos bancos de dados vai depender se os casos clínicos avaliados são raros, se são problemas relacionados a intervenção, prevenção de alguma doença, teste diagnóstico e avaliação da qualidade de vida. Estes parâmetros poderão guiar o leitor na busca eletrônica, assim como na metodologia que pode ser aprendida em sala de aula. A avaliação do aluno em relação ao número de participantes das pesquisas, período e local em que o estudo foi realizado e como os autores chegaram a seus resultados é uma ferramenta que pode auxiliar na avaliação de uma evidência científica de qualidade ${ }^{7}$.

\section{CONCLUSÃO}

No internato de Medicina, os estudantes constroem conhecimentos por meio de metodologias ativas de ensino-aprendizagem, responsabilizando-se progressivamente pelo cuidado nos diversos níveis de atenção à saúde. $\mathrm{O}$ conhecimento de como avaliar um artigo científico, sua construção e seu desenho contribui para que o estudante de graduação possa selecionar melhor a literatura que vai ajudá-lo tanto em seu desenvolvimento crítico como em sua prática médica diária. $\mathrm{O}$ uso de uma ferramenta importante como a leitura de artigos científicos publicados em periódicos de qualidade contribui para o desenvolvimento do raciocínio clínico, que, somado a uma boa prática médica, terá grande impacto na qualidade da assistência clínica.

\section{REFERÊNCIAS}

1. Lopes AA. Medicina Baseada em Evidências: a arte de aplicar o conhecimento científico na prática clínica. Rev Ass Med Brasil 2000; 46(3): 285-8 285.

2. Crato AN, Vidal LF, Bernardino PA, Ribeiro HC, Zarzar PMPA, Paiva SM, Pordeus IA. Como Realizaruma Análise Crítica de um Artigo Científico; Arquivos em Odontologia. Jan/mar 2004; 40(1): 001-110.

3. Martins J. Sousa LM, Oliveira AS. Recomendações do enunciado CONSORT para o relato de estudos clínicos controlados e randomizados; Medicina (Ribeirão Preto) 2009; 42(1): 9-21.

4. DuPrel JB, Röhrig B,Blettner M.Critical Appraisal of Scientific Articles; Deutsches Ärzteblatt International Dtsch ArzteblInt. 2009; 106(7): 100-5.

5. Amoretti R. A Educação Médica diante das Necessidades Sociais em Saúde; Revista Brasileira de Educação Médica. Rio de Janeiro. Maio/ago 2005; 29 (2): 137-46.

6. Costa NMSC. Docência no ensino médico: por que é tão difícil mudar? Rev. bras. educ. med. [online]. 2007, vol.31 (1): 21-30.

7. Kara-Junior N. O valor da análise crítica da literatura para a atualização médica continuada; Rev. bras. oftalmol. Rio de Janeiro May/Jun 2013; 72 (3): 155-56.

\section{CONTRIBUIÇÃO DOS AUTORES:}

Francisco Eduardo Silva: concepção e delineamento do estudo, revisão bibliográfica, redação do artigo, indicação de literatura e revisão crítica.

\section{CONFLITO DE INTERESSES}

não há

\section{ENDEREÇO PARA CORRESPONDÊNCIA}

Avenida Sete de Setembro, 318/1101 - Icaraí - Niterói - RJ 24230-252 